\title{
International Journal of Central Nervous System Disease
}

(ISSN:2637-3823)

\section{Demographic and Clinical Characteristics of Epilepsy in Childhood: A Prospective Cross Sectional Study from Sudan}

Mohamed Osman Omer Dablouk ${ }^{1}$, Amin Alagib Mohamed Musa ${ }^{1}$, Ahlam Abdulrahman Hamed $^{2}$, Rabih Brair Rabih ${ }^{1}$, Khalid Awad Mohamed ${ }^{3^{*}}$, Mamoun Mohamed Ali Homeida ${ }^{1}$

${ }^{1}$ University of Medical Science and Technology. ${ }^{2}$ University of Khartoum. ${ }^{3}$ Weil Cornel Medicine QA.

\section{ABSTRACT}

Background: Epilepsy is a common neurological disorder affecting around $1 \%$ of children. The incidence in Sudan is likely to be higher given the high rate of genetic disorders related to

The incidence of idiopathic epileconsanguity and acquired disorders caused by CNS infection and birth injury. Epilepsy can be idiopathic or non-idiopathic, symptomatic epilepsy indicates a known cause while cryptogenic epilepsy implies a presumed unknown cause. Objective: To understand the demographic and clinical characteristics of epilepsy in children in the setting of a tertiary epilepsy clinic in Khartoum, Sudan. Methods: This is a hospital-based cross sectional study recruiting patients with epilepsy attending a tertiary pediatric neurology clinic. All patients attending the clinic in the 6 month period were included. Data was collected by medical students and was analyzed on IBM SPSS Version 20.0 in a descriptive fashion. Results: 284 Children were recruited. The mean age for children with epilepsy was 5.74 (range 0-12 Years). Females constituted 59.9\%; while $94.7 \%$ of patients were of low socioeconomic status. The most common type of seizures found were generalized tonic clinic at 51.8\% (147/284), followed by focal seizures at $21.1 \%(60 / 284)$. $71 \%$ of our patients (201/284) had no identifiable cause (idiopathic), while $29 \%$ were non-idiopathic. Of the non-idiopathic group; $41 \%$ were due to congenital anomalies, followed by HIE at $24.1 \%$, infections at $16.9 \%$ trauma at $10.8 \%$ and tumors at $7.2 \%$. Only $18.3 \%(52 / 284)$ had a positive family history of epilepsy.

Conclusions: There appears to be a higher incidence of epilepsy in Children with low socio - economic status with a slight female predominance.

psy appears to be similar to international figures.

We recommend broader community based and long-term studies for better understanding of epilepsy in Sudan.

*Correspondence to Author:

Dr Khalid Mohamed

Senior Attending Physician, Sidra Medicine, PO Box 26999, Doha, Qatar.

How to cite this article:

Mohamed Osman Omer Dablouk, Amin Alagib Mohamed Musa, Ahlam Abdulrahman Hamed, Rabih Brair Rabih, Khalid Awad Mohamed Mamoun Mohamed Ali Homeida. Demographic and Clinical Characteristics of Epilepsy in Childhood: A Prospective Cross Sectional Study from Sudan.International Journal of Central Nervous System Disease, $2021 ; 2: 6$

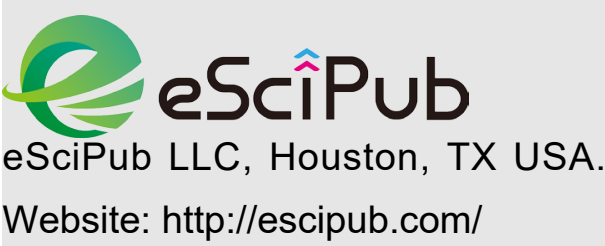




\section{Introduction}

Epilepsy is defined as recurrent epileptic seizures. The lifelong incidence of single seizures is around $5-10 \%$. Of those, roughly $50 \%$ develop a second episode of seizure ${ }^{[1,2]}$. Epilepsy has a high incidence in childhood that declines with age and it is estimated that around $1 \%$ of children worldwide have a diagnosis of epilepsy. Most patients with epilepsy have convulsive seizures and those are broadly divided into generalized and foal onset seizures ${ }^{[3]}$.

A small group of children develop an epileptic encephalopathy, characterized by refectory seizures and developmental regression, those disorders often have a genetic origin and the lack of appropriate genetic testing and counselling adds to the burden of managing those children in resource limited countries like Sudan ${ }^{[4]}$.

Genetic factors including the effect of inter-marriage have been studied in epilepsy by researchers and there is a positive correlation between the burden of genetic disorders and the incidence of epilepsy, in particular non-idiopathic epilepsy and epileptic encephalopathy ${ }^{[5]}$.

Around $70 \%$ of all patients with epilepsy are controlled on medications or go on to have a longterm remission while around $30 \%$ become refractory to treatment or difficult to control and a small minority require non-drug treatment including dietary treatment and surgical intervention.

Epilepsy carries significant morbidity which includes the burden of seizures, the educational and learning effects as well as the stigma associated with the condition especially in societies where illiteracy and poverty limit access to good medical care and information. Often patients with epilepsy are treated by traditional healers and considered to have a mental illness or be affected by evil-spirits.

The effect of epilepsy in children is compounded by the fact that many children with epilepsy have co-morbidities such as physical disability attentional problems and psychiatric and behavioral difficulties, in developing counties such as Sudan those are usually under-diagnosed and combined with the access issues contribute to the lower quality of life experienced by those chil- dren.

There are several studies that defined the incidence and the clinical presentation of epilepsy in developing countries including studies from Africa ${ }^{[6]}$.

Several studies to understand the disorder in children in Sudan have been published in recent years; in particular since the tertiary service was initiated; studies on the prevalence of epilepsy, classification and management and the quality of life in children with the disorder were published recently by the tertiary pediatric neurology group. While the epidemiological work undertaken before was focused on the community; this prospective study intends to define the demographic characteristics and the clinical features of children presenting to the tertiary pediatric epilepsy clinic ${ }^{[7,8]}$.

\section{Methods \\ Study design}

This is a hospital based cross sectional prospective study.

\section{Study Setup}

The study was carried out in the pediatric neurology tertiary clinic in Soba University Hospital, this is a referral service that was founded in 2012 to provide tertiary pediatric neurology care for the children in Khartoum and beyond; referrals are accepted from all states.

\section{Study populations}

All consecutive children with the diagnosis of epilepsy were approached to participate in the study. All those who consented were enrolled.

Families who declined to participate were excluded as all individuals over the age of 18 .

\section{Data collection}

Detailed questionnaire with aspects in the history and family history and details about the socio-economic status of the patient including parental income and housing status were collected by the study team. Socioeconomic status was determined by the family income, access to housing and access to schooling.

Clinical examination was conducted by a pediatric neurology consultant while details of the imaging were collected form the patient's chart, all other information were collected form the chart 
and verified either with the family or the treating physician.

Statistical analysis: Undertaken using the SSPS version 20.

\section{Results \\ Demographics}

The mean age for children with epilepsy between the age of 1-12 was found to be 5.74 (Table 1).

Most children with epilepsy (95\%) came from low socioeconomic status with poor housing and low income, while there was a slight female gender prevalence

The majority affected by epilepsy were from Khartoum City at 53.2\% (151/284) followed by Khartoum North at 25\% (71/284), followed by Omdurman at 21.8\% (62/284) (Table 1).

This indicates the current location of the patients although many of them have travelled from the states to be seen in the tertiary level service.

Table (1): shows the demographic distribution of participants

\begin{tabular}{cccc}
\hline \multirow{2}{*}{ Gender } & Male & 114 & 40.1 \\
& Female & 170 & 59.9 \\
Residence & & & \\
& Khartoum & 151 & 53.2 \\
& Omdurman & 62 & 21.8 \\
& Bahri & 71 & 25 \\
Socioeconomic status & & & \\
& Low & 269 & 94.7 \\
& Moderate & 15 & 5.3 \\
\hline
\end{tabular}

Seizure type etiology and epilepsy Syndromes

Most children presented with generalized seizures, while $21 \%$ presented with focal seizures. The etiology was reported to be idiopathic in around $70 \%$ and either symptomatic or cryptogenic in $30 \%$

We did not have access to all EEG records and it was difficult to classify the epilepsy syndrome, however, in children with idiopathic epilepsy ,100 children (50\%) had a history suggestive of benign focal childhood epilepsy based on the clinica-
I picture and the normal development.

Of the non-idiopathic group, congenital and genetic disorders (41\%) and Hypoxic injury at birth (24\%) accounted for two thirds of the total.

\section{Familial cases}

Only 52 (18\%) children with epilepsy reported a close family history of epilepsy in a parent or sibling.

We did not have genetic results for the patients as the resources and the cost of the tests were beyond the reach of most patients in this clinic.

Table (2): Seizure type and family history as well as idiopathic and symptomatic

\begin{tabular}{cccc}
\hline & & Frequency & Percent \\
Type of Seizure & Focal & 60 & 21.1 \\
& Generalized Tonic Clonic & 147 & 51.8 \\
& Myoclonic & 28 & 9.9 \\
& Absence & 39 & 13.7 \\
Cause & Others & 10 & 3.5 \\
& Idiopathic & 201 & 70.8 \\
& Non-Idiopathic/sympto- & 83 & 29.2 \\
& matic & & \\
Family history & Positive & 52 & 18.3 \\
\hline
\end{tabular}




$\begin{array}{ccc}\text { Congenital Anomalies } & 34 & 41 \\ \text { Neoplasm } & 6 & 7.2 \\ \text { HIE } & 20 & 24.1 \\ \text { Infection } & 14 & 16.9 \\ \text { Trauma } & 9 & 10.8\end{array}$

\section{Discussion}

Our study is prospective and was undertaken to understand the demographic and clinical presentation of epilepsy in pediatrics in Sudan, this is a hospital-based study and compliments work done before to study the prevalence in the community $[7,8,11]$.

The study recruited a large number of patients attending this tertiary clinic and evaluated the epidemiology of the disorder. A recent study published by Mohamed et al (2019) ${ }^{[8]}$ evaluated the presentation of epilepsy in a smaller sample of school children while this work reviews the epidemiology in the children who have already been referred to the tertiary pediatric neurology service.

Mohammed et al investigated the prevalence of epilepsy in Khartoum schools by doing a large epidemiological study; they estimated the prevalence to be around 4/1000 which is much higher than the prevalence identified in an earlier study on $0.9 / 1000$ in $1983^{[12]}$. Both studies would have not included pre-school children and children with early infantile epilepsies in which children die early or have severe disabilities and would not be in the school system ${ }^{[7]}$.

The current study includes younger children who have severe epilepsy and pre-school children as it is conducted in the hospital clinic setting as well as school age children. The prevalence of epilepsy is therefore likely to be much higher than the estimated prevalence in the epidemiological studies and would probably be similar to the international figure of around 0.5 to $1 \%{ }^{[13]}$. Epilepsy is a condition with significant morbidity and is associated with a high level of social stigma and psychological suffering especially in Sudan [14].

Surprisingly, girls were more affected than boys in this study group and this may be related to the fact that girls are more likely to attend clinics while some of the older boys may already be in the job market or prefer not to attend the clinic for social reasons in those families and this may well relate to the social and economic factors that will be discussed in detail.

Stigma related to the diagnosis of epilepsy is a very strong factor that adds to the morbidity and suffering of those children and also limits the management opportunities as children may not be brought to the epilepsy clinic or ignore the symptoms because of the stigma that is associated with the condition. This has been discussed by other authors both in developed countries ${ }^{[15]}$ and developing countries ${ }^{[16]}$.

In our study the majority of children with epilepsy come from families with low socioeconomic status, this is probably multifactorial; the availability of access to free healthcare in our tertiary university hospital might have attracted poorer families to attend this clinic especially that most of the families are from Khartoum city, however it is also noted that epilepsy is more common in patients who come from low socioeconomic backgrounds. This has been reported by researchers from Sweden ${ }^{[17]}$ and Zambia ${ }^{[18]}$. Our work confirms the association between epilepsy and low socioeconomic status.

\section{Conclusions}

The epidemiology of epilepsy in childhood in Sudan has been explored before, this prospective study confirms the high prevalence of epilepsy and the association with low socioeconomic status. The percentage of patients with Idiopathic epilepsy is consistent with the international reported incidence.

Further research is needed to study the social stigma and psychosocial effects of epilepsy on 
children and young people in Sudan.

\section{References}

[1] Berg, AT (2008). Risk of recurrence after a first unprovoked seizure. Epilepsia, 49(Suppl. 1):1318, 2008.

[2] Lois G Kim ${ }^{1}$, Tony L Johnson, Anthony G Marson, David W Chadwick Prediction of risk of seizure recurrence after a single seizure and early epilepsy: further results from the MESS trial. Lancet Neurol. 2006 Apr;5(4):317-22

[3] Fisher R, van Emde Boas W, Blume W, Elger C, Genton P, Lee P, Engel J (2005). "Epileptic seizures and epilepsy: definitions proposed by the International League against Epilepsy (ILAE) and the International Bureau for Epilepsy (IBE)". Epilepsia. 2005 Apr;46(4):470-2

[4] Mastrangelo M, Leuzzi V. Genes of early-onset epileptic encephalopathies: from genotype to phenotype. Pediatr Neurol. 2012 Jan. 46(1):24-31.

[5] Hunza Khan, Abeer Mohamed, Zina-Al-Sakini, Kish war Zulfiquar, Ali Sohail, Rizwana B Shaikh, Shatha Al Sharbatti, Elsheba Mathew; A case control study: Consanguinity, family history and risk of epilepsy; Gulf Medical Journal 2012; (1) 32 - 36

[6] Anthony K Ngugi $\stackrel{1}{ }$, Symon M Kariuki $\stackrel{2}{2}$, Josemir W Sander Prevalence and potential causes of epilepsy in Nigeria Epilepsy Res2014 Oct;108(8): 1480

[7] Mohamed I, Elseed M, Hamed A et al Prevalence of epilepsy in 74,949 school children in Khartoum State, Sudan Paediatrics and international child health 2017: 37(3):1-5

[8] Mohamed I, Elseed M, Mohamed S et al Classification and management of epilepsy and epileptic syndromes in a cohort of 202 school children- a 2 year follow up study- Sudan BMC Neurology volume 19, Article number: 290 (2019)

[9] Benamer HT, Grosset DG. A systematic review of the epidemiology of epilepsy in Arab countries. Epilepsia. 2009; 50(10):2301-2304.

[10] Wanigasinghe J, Arambepola C, Murugupillai R,
Chang T. Age, sex and ethnic differentials in the prevalence and control of epilepsy among Sri Lankan children: a population-based study. BMJ Paediatr Open. 2019;3(1)

[11] Hamed AA, Elseed MA, Mohammed IN. Epidemiology of epilepsy among school children in Khartoum State-2014. Sudanese Journal of Paediatrics. $2015 ; 15(1): 118-119$

[12] Younis YO. Epidemiology of epilepsy among school populations in Khartoum Province, Sudan. J Trop Med Hyg 1983; 86:213-216

[13]Kari Modalsli Aaberg, Nina Gunnes, Inger Johanne Bakken, Camilla Lund Søraas, Aleksander Berntsen, Per Magnus, Morten I. Lossius, Camilla Stoltenberg, Richard Chin and Pål Suré Incidence and Prevalence of Childhood Epilepsy: A Nationwide Cohort Study Pediatrics May 2017, 139 (5) e20163908;

[14] Muwada Bashir, Shahd Abdalla, Ngwayu Nkfusai, Frankline Nsai, Rosaline Cumber, Joyce Gwegweni, samuel Cumber Stigma on epileptic patients attending the outpatient clinic ofSoba University Hospital and the National Center for Neurological Science (NCNS), Khartoum, Sudan Pan African Medical Journal 2019;32:93

[15] Joanne taylor Gus A.Baker, Ann Jacoby Levels of epilepsy stigma in an incident population and associated factors Epilepsy \& Behavior Volume 21, Issue 3, July 2011, Pages 255-260

[16] RoyGretchen, L.Birbeck Epilepsy-associated stigma in sub-Saharan Africa: The social landscape of a disease Epilepsy \& Behavior Volume 7, Issue 1, August 2005, Pages 68-73.

[17]Xinjun Li, Jan Sundquist and Kristina Sundquist, Socioeconomic and occupational risk factors for epilepsy: a nationwide epidemiological study in Sweden Seizure. 2008 Apr; 17(3): 254-260

[18]Birbeck G, Chomba E, Atadzhanov M, Mbewe E, Haworth A The social and economic impact of epilepsy in Zambia: a cross-sectional study. The Lancet. Neurology, Jan 2007, 6(1):39-44

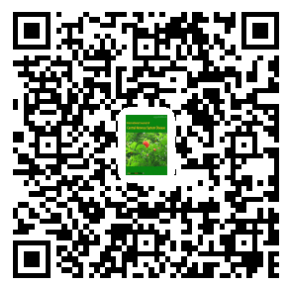

